\title{
Test of internal-conversion theory with a measurement in ${ }^{111} \mathrm{Cd}$
}

\author{
N. Nica ${ }^{1, a}$, J.C. Hardy ${ }^{1}$, V.E. Iacob $^{1}$, T.A. Werke ${ }^{1}$, C.M. Folden $\mathrm{III}^{1}$, L. Pineda ${ }^{1}$, and M.B. Trzhaskovskaya ${ }^{2}$ \\ 1 Cyclotron Institute, Texas A\&M University, College Station, Texas 77843, USA \\ 2 Petersburg Nuclear Physics Institute, Gatchina 188300, Russia
}

\begin{abstract}
We have measured the $K$-shell and total internal conversion coefficients for the $150.8-\mathrm{keV} E 3$ transition in ${ }^{111} \mathrm{Cd}: \alpha_{K}=1.449(18)$ and $\alpha_{T}=2.217(26)$ respectively. The $\alpha_{K}$ value agrees well with DiracFock calculations, in which the effect of the $K$-shell atomic vacancy is included. It is consistent with our previous precise measurements of $\alpha_{K}$ values, which cover a range of atomic numbers, and extends that range down to $Z=48$. The $\alpha_{T}$ measurement, however, disagrees by about two standard deviations from the calculated $\alpha_{T}$ value, whether the atomic vacancy is included or not.
\end{abstract}

\section{Introduction}

The internal conversion coefficient (ICC) is a critical property of each nuclear electromagnetic transition; it is defined as the ratio of electron to $\gamma$-ray emission. As the origin of the emitted electrons can in general be any one of the atomic shells, one can denote the ICCs corresponding to each shell by $\alpha_{K}, \alpha_{L}, \alpha_{M}$, and so on; and their sum by $\alpha_{T}$, the total ICC.

The ICCs are essential ingredients in the construction of nuclear decay schemes: They are required to determine total transition intensities, and they can be used in the assignment of spins and parities. Moreover, internal conversion plays an important role in medical, pharmaceutical and environmental applications of nuclear science. Thus it is important to the nuclear data community to have access to precise measurements of ICCs and to have the ability to calculate ICCs reliably where measurements do not exist.

For several decades, most ICC values appearing in nuclear databases were obtained from tabulated calculations, partly because the ICCs for most transitions had never been measured, and partly because the accuracy and precision of the values that had been measured were generally rather modest, being in the 5-10\% range. In fact, this precision is quite adequate if the goal is to determine spins and parities based on the multipolarity of a transition, but it is far from adequate if precise transition intensities are sought. All ICC databases were elaborated using the Dirac-Slater method where the exchange between electrons was taken into account approximately. The agreement between theory and experiment was considered as satisfactory despite the fact that theoretical ICCs for high-multipolarity transitions systematically turned out to be $2-3 \%$ larger than experimental values.

About 15 years ago, this situation was seen to be unsatisfactory and Raman et al. [1] published a careful study that compared the available tables of calculated ICCs with one another and with a compilation of all

a e-mail: nica@comp.tamu.edu published measurements of ICC values. By then the ICC calculations based on the Dirac-Fock method were developed [2] where the exchange was included exactly between bound electrons as well as between bound and continuum ones. Extensive comparison of new theoretical ICC with measured values for 100 transitions having experimental uncertainties $\leq 5 \%$ has showed that the Dirac-Fock calculations reproduce experimental values, on average, at the $\lesssim 1.5 \%$ level. However since the measurements themselves were far from precise, it was difficult to draw a very firm conclusion. In particular, one ambiguity was made manifest: How should the atomic vacancy created by the ejected electron be treated? Some calculations took account of the vacancy; other did not. With about 20 ICCs measured with a precision of $2 \%$ or better, Raman et al. concluded that Dirac-Fock calculations by Band et al. [3] with the vacancy ignored agreed statistically better with data than a similar calculation by the same authors in which the effect of the vacancy was incorporated. This agreement influenced the National Nuclear Data Center (NNDC) and the Nuclear Structure and Decay Data international network (NSDD) to adopt the former calculations in the Evaluated Nuclear Structure Data File (ENSDF), the most comprehensive nuclear database available, which is used for the Nuclear Data Sheets published by Elsevier.

From a physics perspective, this choice is unsupportable. The lifetimes of atomic vacancies are well known to be between $10^{-15}$ and $10^{-17} \mathrm{~s}[4]$; and it is easy to show that this time is longer - usually much longer - than the time it takes for a conversion electron to leave the atom [5]. Surely in the calculation of ICCs the continuum wave function of the ejected electron must be calculated in the field of the ion, not of the neutral atom.

A limitation of the Raman et al. study was the striking lack of precise ICC measurements. Consequently, in 2003 we started a program at Texas A\&M University to measure benchmark ICC values, with the goal to provide a more precise and reliable data set, one particularly geared to addressing the atomic-vacancy issue. So far, we have published a series of papers [5-13] that report 


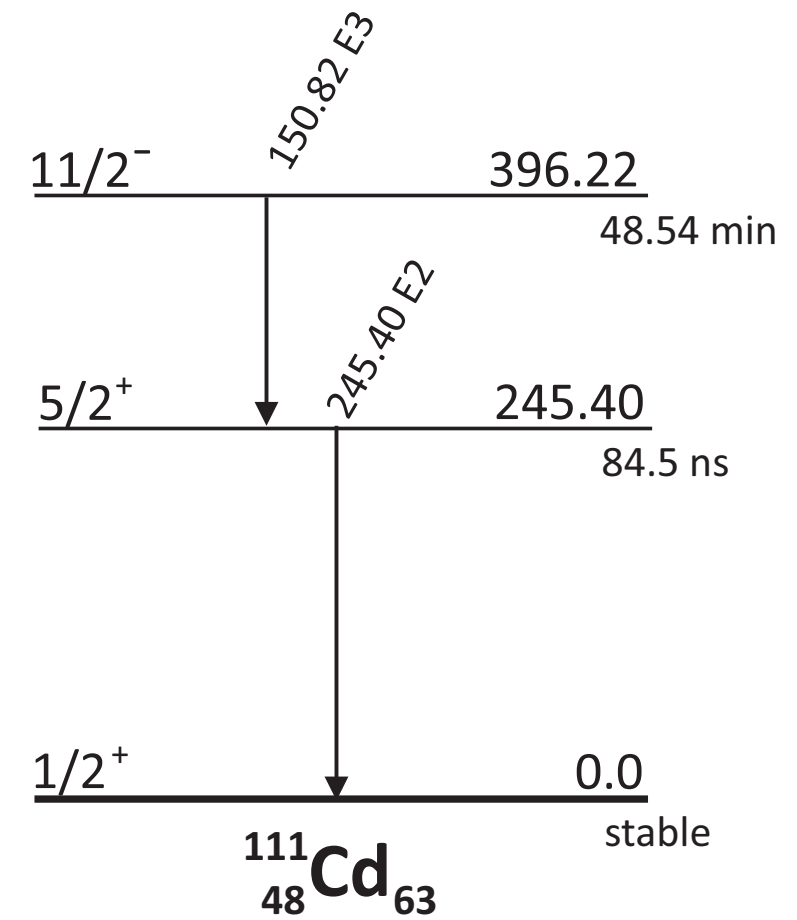

Figure 1. Decay scheme for the 48.5 -min isomer in ${ }^{111} \mathrm{Cd}$. The data are taken from Ref. [14].

ICC values in 6 nuclei, covering the range $48<\mathrm{Z}<78$, with a precision of $2 \%$ or better. Here we outline our measurement of the $150.8-\mathrm{keV}$ E3 transition in ${ }^{111} \mathrm{Cd}$, which extended the range of nuclei down to $Z=48$. We report both $\alpha_{K}$ and $\alpha_{T}$ values to precisions of about $1.2 \%$ [13]. An overview of results from our whole series of measurements is also presented, clearly demonstrating that the atomic vacancy must be included in ICC calculations.

\section{Experimental method overview}

The method we have employed in our previous measurements depends not upon detecting the conversion electrons but rather the characteristic $K$ x rays emitted following the departure of $K$-shell conversion electrons. Since both the $K \mathrm{x}$ rays and the $\gamma$ rays from a given transition can be recorded in same HPGe-detector spectrum, their relative intensities can be determined more reliably than if separate detectors had to be used for the electron and the $\gamma$ ray. This method is suitable for decay schemes dominated by a single transition, in which case the $K$-shell ICC is derived according to the formula:

$$
\alpha_{K}=\frac{N_{K}}{N_{\gamma}} \cdot \frac{\epsilon_{\gamma}}{\epsilon_{K}} \cdot \frac{1}{\omega_{K}},
$$

where $N_{K}$ and $N_{\gamma}$ are the total numbers of observed $K \mathrm{x}$ rays and $\gamma$ rays, respectively; $\epsilon_{K}$ and $\epsilon_{\gamma}$ are the corresponding photopeak detection efficiencies; and $\omega_{K}$ is the fluorescence yield, which is the fraction of $K$-shell vacancies that result in the emission of a $K$ x ray. For cadmium we use $\omega_{K}=0.842(4)$, which is taken from the evaluation of Schönfeld and Janssen [15], the same source that we used for all our previous measurements. It provides reliable $\omega_{K}$ values with sub-percent precision, in this case $\pm 0.5 \%$.
The decay scheme of the 48.5-min isomer in ${ }^{111} \mathrm{Cd}$, appearing in Fig. 1, shows a cascade of two transitions, both of which can convert in the $K$ shell. This does not satisfy the single-transition condition required for Eq. (1), but can be accommodated by a modified version of that equation:

$$
\alpha_{K 150}=\frac{N_{K}}{N_{\gamma 150}} \cdot \frac{\epsilon_{\gamma 150}}{\epsilon_{K}} \cdot \frac{1}{\omega_{K}}-\alpha_{K 245} \cdot \frac{N_{\gamma 245}}{N_{\gamma 150}} \cdot \frac{\epsilon_{\gamma 150}}{\epsilon_{\gamma 245}} .
$$

The numerical subscripts designate the transition - either the $150.8-\mathrm{keV}$ or $245.4-\mathrm{keV}$ one - to which the quantities refer. Evidently $\alpha_{K 150}$, the quantity we seek to determine, now depends on $\alpha_{K 245}$, but since the $245.4-\mathrm{keV}$ transition is pure $E 2$, with a calculated $K$-conversion coefficient about an order of magnitude smaller than $\alpha_{K 150}$, it has very little impact on the precision with which $\alpha_{K 150}$ can be determined.

The presence of a second transition in cascade also allows us to determine $\alpha_{T 150}$ by using the equation:

$$
\left(1+\alpha_{T 150}\right) \cdot \frac{N_{\gamma 150}}{\epsilon_{\gamma 150}}=\left(1+\alpha_{T 245}\right) \cdot \frac{N_{\gamma 245}}{\epsilon_{\gamma 245}} .
$$

Just as we found for the $\alpha_{K}$ values in Eq. (2), here $\alpha_{T 245}$ is much smaller than $\alpha_{T 150}(\sim 0.06 v s . \sim 2.2)$ so the experimental result obtained for $\alpha_{T 150}$ is rather insensitive to the calculated value used for $\alpha_{T 245}$.

Note that in obtaining calculated values for $\alpha_{K 245}$ and $\alpha_{T 245}$ for use in Eqs. (2) and (3) we adopted the average of the values calculated with and without the atomic vacancy as given by the code BrIcc [16] (implemented at http://bricc.anu.edu.au). We also attached an error bar to the averages that encompassed both calculated results. The values we arrived at were

$$
\begin{aligned}
\alpha_{K 245} & =0.05326(17) \\
\alpha_{T 245} & =0.06351(17) .
\end{aligned}
$$

In this way, we ensured that the $\alpha_{K 150}$ and $\alpha_{T 150}$ values obtained from Eqs. (2) and (3) were independent of the treatment of the vacancy for $\alpha_{K 245}$ and $\alpha_{T 245}$.

In applying Eqs. (2) and (3) we also took care that the $N$ values we used incorporated corrections for coincidence summing, including the effect of angular correlation between the two gamma rays.

We used the same HPGe detector as in our previous measurements to measure both the $\gamma$ rays and the $K$ $\mathrm{x}$ rays, a detector whose efficiency has been calibrated thoroughly to sub-percent precision over the energy range from 50 to $3500 \mathrm{keV}$ [17-19]. Precisely measured data were combined with Monte Carlo calculations from the CYLTRAN code [20] to yield a very precise and accurate detector efficiency curve over the whole energy interval. We recently extended the well-calibrated region down to $22.6 \mathrm{keV}$ [12], the average energy of silver $K$ x rays.

For the ${ }^{111} \mathrm{Cd}$ measurement the $\gamma$ rays of interest, at 150.8 and $245.4 \mathrm{keV}$, are within the energy interval of our best calibration, for which our efficiencies are determined to a relative precision of $\pm 0.15 \%$. For the cadmium $K \mathrm{x}$ rays, which lie between 23 and $27 \mathrm{keV}$ in our extended calibration region, we know the detector efficiency to a relative precision of $\pm 1 \%$. 
Table 1. The contributions of identified impurities that affect the cadmium $K$ x-ray peaks.

\begin{tabular}{llcl}
\hline Source & Contaminant & \multicolumn{2}{c}{ Contribution to spectrum (\%) } \\
\cline { 3 - 4 } & & \multicolumn{1}{c}{$\mathrm{S} 1$} & $\mathrm{~S} 2$ \\
\hline${ }^{115} \mathrm{Cd}$ & In $K$ x rays & $1.81(8)$ & $2.71(6)$ \\
${ }^{117} \mathrm{Cd}$ & In $K$ x rays & $0.113(12)$ & $0.125(9)$ \\
${ }^{117} \mathrm{In}$ & $\mathrm{Sn} K$ x rays & $0.18(3)$ & $0.21(3)$ \\
${ }^{117 m} \mathrm{In}$ & In+Sn $K$ x rays & $0.603(9)$ & $0.484(5)$ \\
${ }^{116 m} \mathrm{In}$ & In $K$ x rays & $0.010(2)$ & $0.006(1)$ \\
\hline
\end{tabular}

\section{The ${ }^{111} \mathrm{Cd}$ experiment}

Two sources were prepared - S1 and S2 - from 95.88\%enriched ${ }^{110} \mathrm{CdO}$ electroplated onto a $10-\mu \mathrm{m}$-thick foil of 99.999\% pure aluminum. We produced ${ }^{111} \mathrm{Cd}$ by activating each source, two months apart, with a flux of thermal neutrons of $\sim 7 \times 10^{12} \mathrm{n} /\left(\mathrm{cm}^{2} \mathrm{~s}\right)$ at the TRIGA reactor, located at the Texas A\&M Nuclear Science Center. The initial activity of ${ }^{111 m} \mathrm{Cd}$ was $\sim 80 \mathrm{kBq}$ for each source.

We collected HPGe spectra from both sources using the same detector and source-to-detector distance $(151 \mathrm{~mm})$ as was used in the original efficiency calibration [18]. Our analog-to-digital converter was an Ortec TRUMP $^{T M}-8 \mathrm{k} / 2 \mathrm{k}$ card controlled by MAESTRO ${ }^{T M}$ software. With source S1 we acquired $148 \mathrm{k}$-spectra over a two-week period; with $\mathrm{S} 2$ we acquired 10 spectra in one week. Since ${ }^{111 m} \mathrm{Cd}$ has a half-life of only $49 \mathrm{~min}$, all but the first few spectra recorded in each case were used solely as an aid in identifying contaminant activities. All subsequent analysis for ${ }^{111 m} \mathrm{Cd}$ itself was based on only two spectra - the first 9-min spectrum taken from S1 and the first 15-min spectrum from S2 - in which the ${ }^{111 m} \mathrm{Cd}$ activity was most dominant. It is the results from these two spectra that are reported here.

\section{Data analysis}

The recorded spectra were thoroughly analyzed to identify all radioactive isotopes present in our sources and to determine quantitatively those contaminants affecting the energy regions of interest, around the cadmium $K \mathrm{x}$ rays $(23-27 \mathrm{keV})$ and the $150.8-$ and $245.4-\mathrm{keV} \gamma$-ray photopeaks.

Figure 2 illustrates the analysis: We identified all the peaks including the weakest based on their energies and intensities, by using our efficiency calibration and the electronic databases, ENSDF (at http://www.nndc.bnl.gov) and the Lund Table of Radioactive Isotopes (at http://nucleardata.nuclear. lu.se/toi). In total, we found five weak sources of contamination that affect the cadmium $K$ x ray region. These are listed in Table 1, where their contributions are given as percentages of the total cadmium $K$ x rays recorded. In all, the impurities contribute less than $4 \%$ so their subtraction had very little affect on the precision of our final result. No impurities were found to interfere with either of the $\gamma$-ray peaks of interest.

The peaks were fitted with the GF2 program [21], in a version which allows us to extract the total number of counts above background within selected energy limits. The "total counts" obtained for all three regions of interest are listed in the first column of Table 2. In the case of the $\mathrm{x}$ rays, the row immediately below gives the total number
Table 2. The total number of counts (or areas of the peaks) for ${ }^{111 \mathrm{~m}} \mathrm{Cd} K$ x rays and the 150.8 - and $245.4-\mathrm{keV} \gamma$ rays, followed by corrections and the corrected area-ratios information required to extract the value of $\alpha_{K 150}$.

\begin{tabular}{lll}
\hline Quantity & \multicolumn{2}{c}{ Value } \\
\cline { 2 - 3 } & \multicolumn{1}{c}{$\mathrm{S} 1$} & \multicolumn{1}{c}{$\mathrm{S} 2$} \\
\hline Cd $\left(K_{\alpha}+K_{\beta}\right)$ x rays & & \\
Total counts & $1.979(6) \times 10^{5}$ & $4.695(9) \times 10^{5}$ \\
Impurities & $-5.39(14) \times 10^{3}$ & $-1.66(3) \times 10^{4}$ \\
Lorentzian correction & $+0.12(2) \%$ & $+0.12(2) \%$ \\
Summing correction & $+0.99(6) \%$ & $+0.99(6) \%$ \\
Attenuation correction & $+0.27(2) \%$ & $+0.29(2) \%$ \\
Corrected counts, $N_{K}$ & $1.952(6) \times 10^{5}$ & $4.593(10) \times 10^{5}$ \\
\hline${ }^{111}$ Cd 150.8-keV $\gamma$ ray & & \\
Total counts & $1.303(11) \times 10^{5}$ & $3.064(25) \times 10^{5}$ \\
Summing correction & $+1.29(6) \%$ & $+1.29(6) \%$ \\
Corrected counts, $N_{\gamma 150}$ & $1.320(12) \times 10^{5}$ & $3.104(25) \times 10^{5}$ \\
\hline 111 Cd 245.4-keV $\gamma$ ray & & \\
Total counts & $3.024(22) \times 10^{5}$ & $7.082(45) \times 10^{5}$ \\
Summing correction & $+0.86(3) \%$ & $+0.86(3) \%$ \\
Corrected counts, $N_{\gamma 245}$ & $3.050(22) \times 10^{5}$ & $7.143(45) \times 10^{5}$ \\
\hline$N_{K} / N_{\gamma 150}$ & $1.479(14)$ & $1.480(12)$ \\
$N_{\gamma 245} / N_{\gamma 150}$ & $2.311(27)$ & $2.301(24)$ \\
\hline
\end{tabular}

of impurity counts to be subtracted, as calculated from the percentage breakdowns in Table 1.

For the $\mathrm{x}$-rays, the peak areas thus obtained were further corrected to account for the long tails characteristic of their Lorentzian shapes. For this purpose we used realistic Voigt functions to generate simulation $\mathrm{x}$-ray peaks that were subsequently analyzed with GF2 in the same manner as our actual data. Thus we established the appropriate correction factor $(+0.12 \%)$ to apply to our analysis of real data. It also appears in Table 2 .

Also listed in the table are the summing corrections that account for real coincidences between the two $\gamma$ rays in cascade or between one $\gamma$ ray and a $K$ x ray from the other transition. In either case, the individual signals are lost and are replaced by a single summed-energy signal. The corrections listed in Table 2 effectively add back the lost events to where they belong. The final correction appearing in the table is to compensate for the finite thickness of our samples, which produces more attenuation of the $K$ x rays than of the higher-energy $\gamma$ rays.

The ratios $N_{K} / N_{\gamma 150}$ and $N_{\gamma 245} / N_{\gamma 150}$ that appear in Eqs. (2) and (3) are also listed at the end of Table 2. Their weighted average is:

$$
\begin{aligned}
N_{K} / N_{\gamma 150} & =1.479(10) \\
N_{\gamma 245} / N_{\gamma 150} & =2.305(18) .
\end{aligned}
$$

The remaining quantities required for Eqs. (2) and (3) are the efficiency ratios, $\epsilon_{\gamma 150} / \epsilon_{\gamma 245}$ and $\epsilon_{\gamma 150} / \epsilon_{K}$. The former is straightforwardly obtained from our efficiency curve via CYLTRAN Monte Carlo calculations [18]. The latter is more challenging. Photon scattering by air or by denser materials in the neighborhood of the source and detector results in events appearing in the spectrum at energies below that of each photopeak. This is particularly troublesome for photopeaks below $50 \mathrm{keV}$ because much of this scattered radiation is indistinguishable from the peak itself.

Consequently we follow the same procedure as the one we used in analyzing the decay of ${ }^{119 m} \mathrm{Sn}$ [12] and 


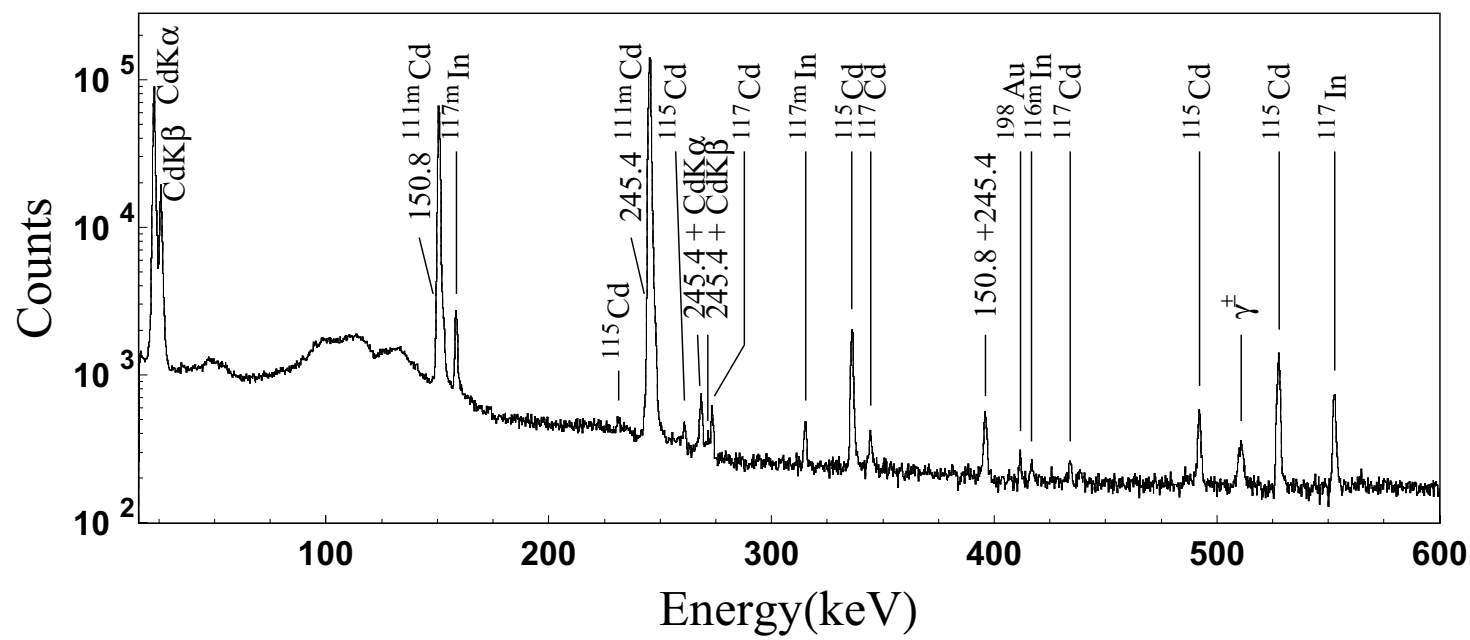

Figure 2. Portion of the $\mathrm{x}$ - and $\gamma$-ray energy spectrum measured for 15 min with source $\mathrm{S} 2$. Room background has been subtracted. The background was recorded before and after the source data were measured; and was normalized to the same live-time. Peaks are labeled by their $\beta$-decay parent; the $\gamma$-ray peaks associated with ${ }^{111 m} \mathrm{Cd}$ decay also have their energies in keV marked.

employ as low-energy calibration the well-known decay of ${ }^{109} \mathrm{Cd}$, which emits $88-\mathrm{keV} \gamma$ rays and silver $K$ x rays. The latter are very close in energy to the cadmium $K \mathrm{x}$ rays observed in the current measurement. Note that we are not distinguishing between $K_{\alpha}$ and $K_{\beta}$ x rays. Because scattering effects are pronounced at these energies we have chosen as before to use only the sum of the $K_{\alpha}$ and $K_{\beta}$ $\mathrm{X}$-ray peaks. For calibration purposes, we consider each sum to be located at the intensity-weighted average energy of the component peaks ${ }^{1}-23.62 \mathrm{keV}$ for cadmium and $22.57 \mathrm{keV}$ for silver.

We obtain the required ratio, $\epsilon_{\gamma 150} / \epsilon_{K 23.6}$ from the following relation:

$$
\frac{\epsilon_{\gamma 150}}{\epsilon_{K 23.6}}=\frac{\epsilon_{\gamma 88.0}}{\epsilon_{K 22.6}} \cdot \frac{\epsilon_{\gamma 150}}{\epsilon_{\gamma 88.0}} \cdot \frac{\epsilon_{K 22.6}}{\epsilon_{K 23.6}},
$$

where we have identified the $K$ x rays by their centroid energy for clarity. We take the ratio $\epsilon_{\gamma 88} / \epsilon_{K 22.6}=1.069(8)$ from our previously reported measurement [12], while the other two ratios on the right hand side were calculated with CYLTRAN: $\epsilon_{\gamma 150} / \epsilon_{\gamma 88.0}=0.8707(13)$ and $\epsilon_{K 22.6} / \epsilon_{K 23.6}=$ $0.9849(9)$. The latter ratio covers an energy span of only $1 \mathrm{keV}$ and is thus very insensitive to scattering effects.

Finally the two efficiency ratios needed for Eqs. (2) and (3) are thus:

$$
\begin{aligned}
& \frac{\epsilon_{\gamma 150}}{\epsilon_{\gamma 245}}=1.3123(14) \\
& \frac{\epsilon_{\gamma 150}}{\epsilon_{K}}=0.917(7) .
\end{aligned}
$$

\section{Results}

Substituting Eqs. (4), (6), and (8) into Eq. (2), we obtain:

$$
\alpha_{K 150}=1.449(18) \text {. }
$$

This result can be compared with the calculated values, 1.425(1) (no vacancy) and 1.451(1) (vacancy included

\footnotetext{
1 To establish the weighting, we used the intensities of the individual x-ray components from Table 7a in Ref. [22].
}

in the so-called frozen orbital, FO, approximation [6]). Our measured value is in very good agreement with the calculated value that includes the atomic vacancy, and is in disagreement with the one that does not.

Substituting Eqs. (5), (6), and (8), into Eq. (3), we obtain:

$$
\alpha_{T 150}=2.217(26)
$$

In this instance the calculated values are 2.257(1) (no vacancy) and 2.284(1) (FO vacancy included). Unlike $\alpha_{K}$, the $\alpha_{T}$ result is lower than both calculations, with the maximum disagreement $(\sim 2.5 \sigma)$ being with the FO calculation.

The $\alpha_{K 150}$ value has only once been measured before, by Németh and Veres [23]. They also used an HPGe detector for both the $\mathrm{x}$ and $\gamma$ rays, and obtained $\alpha_{K 150}=$ $1.29(11)$, which is statistically consistent with our result but is much less precise.

For $\alpha_{T 150}$, three measurements have previously been reported: 2.286(9) [24], 1.76(8) [25], and 1.98(5) [23]. The first measurement used a $\mathrm{NaI}(\mathrm{Tl})$ scintillator; the other two, an HPGe detector. Raman et al. [1] included all of them in their survey, adopting 2.12(11) for $\alpha_{T 150}$. None of the three previous measurements agree with one another or with our new result; however our result does agree with the value adopted by Raman et al. but is much more precise.

Why do both types of calculation disagree with our $\alpha_{T}$ result? One possible explanation arises from the fact that the $150.8-\mathrm{keV}$ transition is hindered by a factor of $10^{4}$ relative to the single-particle Weisskopf estimate [2]. This could produce a "penetration" effect associated with the change from transition electromagnetic potentials used for a point nucleus to transition potentials required for a realistic finite-sized nucleus [26]. For strongly hindered transitions, the penetration effect can give rise to nonnegligible nuclear matrix elements in the expressions for the ICCs, which can thus become dependent on nuclear structure details and nuclear transition dynamics.

For $M 1$ and $E 1$ transitions, penetration factors have been calculated (see for example Refs. [26-29]) but it appears that there are no convincing results for E3 transitions. However, despite the lack of direct support, penetration remains the best explanation for 
Table 3. Comparison of the six measured $\alpha_{K}$ values published in Refs. [5-13] with Dirac-Fock theoretical calculations that either ignored the atomic vacancy (the "No vacancy" column) or included via the "frozen orbitals" approximation (the "Vacancy" column). The total $\chi^{2}$ values demonstrate a dramatic preference for the "frozen orbitals" calculations. The uncertainties of the theoretical values are negligible compared with the experimental ones and were omitted.

\begin{tabular}{lllll}
\hline Parent & Transition & Measured $\alpha_{K}$ & \multicolumn{2}{c}{ Calculated $\alpha_{K}$} \\
\cline { 4 - 5 } & Energy(keV) & & No vacancy & Vacancy \\
\hline${ }^{197 m} \mathrm{Pt}$ & $346.5(2)$ & $4.23(7)$ & 4.191 & 4.276 \\
${ }^{193 m} \mathrm{Ir}$ & $80.22(2)$ & $103.0(8)$ & 92.0 & 103.3 \\
${ }^{137 m} \mathrm{Ba}$ & $661.659(3)$ & $0.0915(5)$ & 0.09068 & 0.09148 \\
${ }^{134 m} \mathrm{Cs}$ & $127.502(3)$ & $2.742(15)$ & 2.677 & 2.741 \\
${ }^{119 m} \mathrm{Sn}$ & $65.66(1)$ & $1610(27)$ & 1544 & 1618 \\
${ }^{111 m} \mathrm{Cd}$ & $150.853(15)$ & $1.449(18)$ & 1.425 & 1.451 \\
$\chi^{2}$ & & & 219 & 0.68 \\
\hline
\end{tabular}

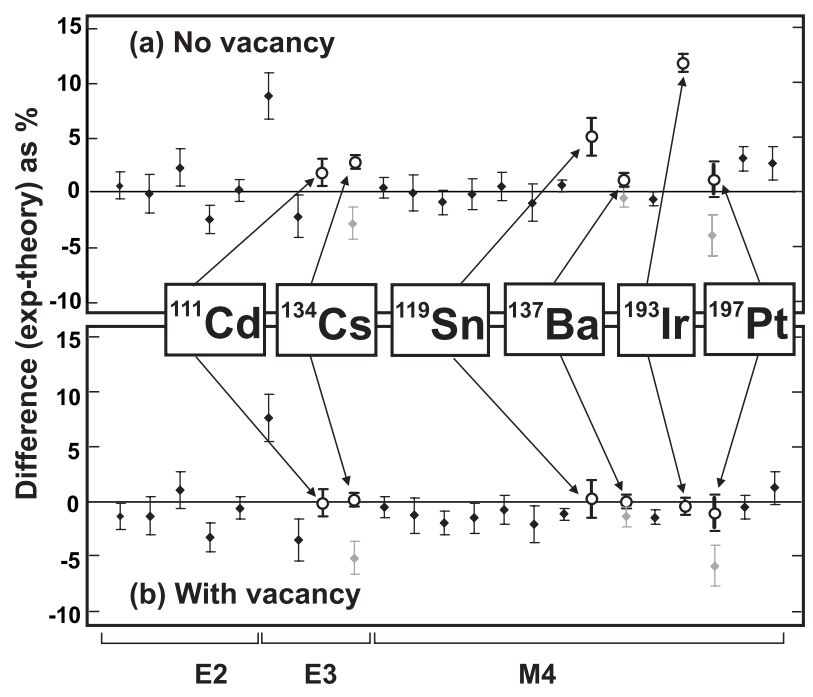

Figure 3. Percentage differences between the measured and calculated ICCs for two DiracFock calculations: one (a) is without the atomic vacancy; the other (b) is with it included in the "frozen orbital" approximation. Shown with solid diamonds are the twenty cases selected by Raman et al. [1] with highest precision - better than $2 \%$. Our $\alpha_{K}$ measured values, labeled on the figure, appear as open circles, with the original Raman et al. values shown in gray. The transition multipolarities $E 2, E 3$, and $M 4$, are indicated at the bottom. We have measured two $E 3$ and four $M 4$ transitions.

the discrepancy we observe here between experiment and theory for $\alpha_{T}$. Moreover, Krpić and Aničin [29] showed (for $M 1$ transitions) that it is possible under particular conditions for the penetration to be "hidden" by a cancellation that can affect one atomic shell but not the others. Hypothetically this could also explain why in our case the $\alpha_{K}$ looks to be unaffected by penetration effects, while $\alpha_{T}$ is significantly reduced.

\section{Current status of precision ICC measurements}

All $\alpha_{K}$ experimental values we published in Refs. [5-13] are compared in Table 3 and Fig. 3 with both types of Dirac-Fock calculations: either excluding the atomic vacancy; or including it through the so-called "frozen orbitals" assumption. In both approximations the electron wave functions in the initial bound state are calculated in the self-consistent field of a neutral atom. The difference occurs in the continuum final states: for the no-vacancy calculation the neutral atom is used again, while for the "frozen orbitals" approximation the continuum wave function is calculated in the ion field constructed from the bound wave functions of the neutral atom; this assumes that there is insufficient time for the atomic orbitals to have rearranged themselves. An inspection of Fig. 3 and the $\chi^{2}$ values in Table 3 clearly shows good agreement with the "frozen orbitals" approximation and disagreement with the no-vacancy assumption. Our current $\alpha_{K}$ result is consistent with all our previous measurements.

Finally, it should be noted that during the course of our series of measurements, Kibédi et al. [16] were prompted to reevaluate the ICCs with our measurements now included, and to develop BrIcc, a new database obtained from the basic code by Band et al. [3]. In conformity with our conclusions, it employed a version of that code which incorporates the vacancy in the "frozen orbital" approximation. The BrIcc database was subsequently adopted by the NNDC and is available online for the determination of ICCs.

We wish to thank the staff of the Texas A\&M Nuclear Science Center for their help in conducting neutron activations. This material is based upon work supported by the U.S. Department of Energy, Office of Science, Office of Nuclear Physics, under Award Number DE-FG03-93ER40773, and by the Robert A. Welch Foundation under Grant No. A-1397.

\section{References}

[1] S. Raman, C.W. Nestor, Jr., A. Ichihara, M.B. Trzhaskovskaya, Phys. Rev. C 66, 044312 (2002); see also the electronic addendum to this paper, the location of which is given in the paper's reference 32

[2] I.M. Band, M.A. Listengarten, M.B. Trzhaskovskaya, Bull. Akad. Sci. USSR, Phys. Ser. 53, 85 (1989)

[3] I.M. Band, M.B. Trzhaskovskaya, C.W. Nestor, Jr., P. Tikkanen, S. Raman, At. Data Nucl. Data Tables 81, 1 (2002)

[4] O. Keski-Rahkonen, M.O. Krause, At. Data Nucl. Data Tables 14,139 (1974)

[5] J.C. Hardy, N. Nica, V.E. Iacob, S. Miller, M. Maguire, M.B. Trzhaskovskaya Appl. Rad and Isot. 87, 87 (2014)

[6] N. Nica, J.C. Hardy, V.E. Iacob, S. Raman, C.W. Nestor Jr., M.B. Trzhaskovskaya, Phys. Rev. C 70, 054305 (2004)

[7] N. Nica, J.C. Hardy, V.E. Iacob, J.R. Montague, M.B. Trzhaskovskaya, Phys. Rev. C 71, 054320 (2005)

[8] N. Nica, J.C. Hardy, V.E. Iacob, W.E. Rockwell, M.B. Trzhaskovskaya, Phys. Rev. C 75, 024308 (2007)

[9] N. Nica, J.C. Hardy, V.E. Iacob, C. Balonek, M.B. Trzhaskovskaya, Phys. Rev. C 77, 034306 (2008)

[10] J.C. Hardy, N. Nica, V.E. Iacob, C. Balonek, M.B. Trzhaskovskaya, Appl. Rad. Isot. 66, 701 (2008)

[11] N. Nica, J.C. Hardy, V.E. Iacob, J. Goodwin, C. Balonek, M. Hernberg, J. Nolan, M.B. Trzhaskovskaya, Phys. Rev. C 80, 064314 (2009) 
[12] N. Nica, J.C. Hardy, V.E. Iacob, M. Bencomo, V. Horvat, H.I. Park, M. Maguire, S. Miller, M.B. Trzhaskovskaya, Phys. Rev. C 89, 014303 (2014)

[13] N. Nica, J.C. Hardy, V.E. Iacob, T.A. Werke, C.M. Folden, L. Pineda, M.B. Trzhaskovskaya Phys. Rev. C 93, 034305 (2016)

[14] J. Blachot, Nucl. Data Sheets 110, 1239 (2009)

[15] E. Schönfeld, H. Janssen, Nucl. Instrum. Methods Phys. Res. A 369, 527 (1996)

[16] T. Kibédi, T.W. Burrows, M.B. Trzhaskovskaya, P.M. Davidson, C.W. Nestor Jr., Nucl. Instrum. Meth. in Phys. Res. A 589, 202 (2008)

[17] J.C. Hardy, V.E. Iacob, M. Sanchez-Vega, R.T. Effinger, P. Lipnik, V.E. Mayes, D.K. Willis, R.G. Helmer, Appl. Radiat. Isot. 56, 65 (2002)

[18] R.G. Helmer, J.C. Hardy, V.E. Iacob, M. SanchezVega, R.G. Neilson, J. Nelson, Nucl. Instrum. Methods Phys. Res. A 511, 360 (2003)

[19] R.G. Helmer, N. Nica, J.C. Hardy, V.E. Iacob, Appl. Radiat. Isot. 60, 173 (2004)
[20] J.A. Halbleib, R.P. Kemsek, T.A. Melhorn, G.D. Valdez, S.M. Seltzer, M.J. Berger, Report SAND9116734, Sandia National Labs (1992)

[21] D. Radford, http://radware.phy.ornl.gov/ main.html and private communication

[22] R.B. Firestone, Table of Isotopes, ed. V.S. Shirley (John Wiley \& Sons Inc., New York, 1996) p. F-44

[23] Zs. Németh and Á. Veres, Phys. Rev. C 35, 2294 (1987)

[24] D.C. Lu, Phys. Rev. 119, 286 (1960)

[25] Ch. Suranarayama, N. Venkateswara Rao, S. Bhuloka Reddy, G. Satyanarayana, D.L. Sastry, J. Phys. Soc. Japan 54, 4539 (1985)

[26] M.A. Listengarten, Anomalous Internal Conversion Coefficients in Electromagnetic Transitions in Atomic Nuclei, in Modern Methods in Nuclear Spectroscopy, ed. B. S. Djelepov (Nauka, Leningrad, USSR, 1985) p. 142 (in Russian)

[27] G. Kramer, S.G. Nilsson, Nucl. Phys. 35, 273 (1962)

[28] J. Le Tourneux, Nucl. Phys. 74, 172 (1965)

[29] D.K. Krpić, I.V. Aničin, Phys. Rev. C 9, 660 (1974) 